\title{
IDENTIFICATION OF MICROCRAKING PROCESS IN FRACTURE PROCESS ZONE BY AE
}

\author{
M. Ohtsu, \\ Graduate School of Science and Technology, Kumamoto University, 2-39-1 Kurokami, Kumamoto 860-8555, Japan.
}

\section{ABSTRACT}

Microcraking process in concrete is readily monitored by acoustic emission (AE) technique. Crack kinematics associated with nucleation of the fracture process zone are identified by AE-SiGMA procedure, by which crack locations, crack types and crack orientation are determined from the moment tensor analysis of AE waveforms.

By applying SiGMA, microcracking process in the process zone is investigated in a notched specimen of concrete. A relation between the damage variable in damage mechanics and the moment tensor is derived. The damage evolution in the fracture process zone is estimated under off-center bending. It is found that the damage evolve gradually in mode I failure along with mode-II failure and mixed-mode. Since the direction of crack extension can be derived from the eigenvectors of the moment tensor, the concept of the maximum circumferential stress is applied to estimate the normalized stress intensity factors. In the beginning of microcraking process, mode I failure is dominant. Then, mode II cracks are observed in the final stage. This result in SiGMA analysis is confirmed by BEM (boundary element method) analysis.

\section{INTRODUCTION}

The generalized theory of acoustic emission (AE) was established on the basis of elastodynamics (Ohtsu and Ono [1]). Although AE waves due to microcracking are discussed in concrete, theoretical treatment is associated with elastic waves in a homogeneous medium (Ohtsu [2]). This is because elastodynamic properties of material constituents are physically dependent on the relation between the wavelengths and the characteristic dimensions of heterogeneity. In the case that the wavelengths are even larger than the sizes of heterogeneous inclusions, the effect of heterogeneity is inconsequent. This is the case of concrete, if the sizes of specimens are large enough compared with the wavelengths.

Crack kinematics of AE source are defined by a crack motion vector (Burgers vector) and a normal vector to a crack plane. These two vectors could leads to the moment tensor analysis (Ohtsu et al. [3]). From the moment tensor, quantitative information on crack kinematics of crack locations, crack types and crack orientations is obtained. In order to determine moment tensor components from AE waveforms, SiGMA procedure (simplified Green's functions for moment tensor analysis) is developed (Ohtsu [4]). AE sources are classified into tensile cracks and shear cracks by applying the eigenvalue analysis to the moment tensor, and the direction of crack motion is derived from the eigenvectors.

In this paper, a relation between the damage variable in damage mechanics and the moment tensor is derived. Then, the damage evolution in the fracture process zone of a notched concrete beam is estimated under off-center bending. Since the direction of crack extension can be derived from the eigenvectors, the normalized stress intensity factors are estimated experimentally.

\section{MOMENT TENSOR AND DAMAGE EVOLUTION}

An elastodynamic solution of wave motion $\mathbf{u}(\mathbf{x}, \mathbf{t})$ due to cracking is mathematically represented, 


$$
\begin{aligned}
\mathrm{u}_{\mathrm{k}}(\mathbf{x}, \mathrm{t}) & =\int_{\mathrm{F}} \mathrm{G}_{\mathrm{kp}, \mathrm{q}}(\mathbf{x}, \mathbf{y}, \mathrm{t}) \mathrm{C}_{\mathrm{pqij}} \mathrm{n}_{\mathrm{j}} \mathrm{w}_{\mathrm{i}}(\mathbf{y}, \mathrm{t}) \mathrm{dF}=\mathrm{G}_{\mathrm{kp}, \mathrm{q}}(\mathbf{x}, \mathbf{y}, \mathrm{t}) * \mathrm{~S}(\mathrm{t}) \mathrm{C}_{\mathrm{pqij}} \mathrm{n}_{\mathrm{j}} \mathrm{l}_{\mathrm{i}} \int_{\mathrm{F}} \mathrm{b}(\mathbf{y}) \mathrm{dF} \\
& =\mathrm{G}_{\mathrm{kp}, \mathrm{q}}\left(\mathbf{x}, \mathbf{y}, \mathrm{t}, * \mathrm{~S}(\mathrm{t}) \mathrm{C}_{\mathrm{pqij}} \mathrm{n}_{\mathrm{j}} \mathrm{l}_{\mathrm{i}} \Delta \mathrm{V},\right.
\end{aligned}
$$

where $\mathbf{b}(\mathbf{y}, \mathbf{t})$ is the crack motion vector (Burgers vector) and the asterisk symbol * represents the convolution integral in time. $C_{p q j i}$ are the elastic constants, and $G_{i p, q}(\mathbf{x}, \mathbf{y}, t)$ are the spatial derivatives of Green's functions as they imply $\partial \mathrm{G}_{\mathrm{ip}}(\mathbf{x}, \mathbf{y}, \mathrm{t}) / \partial \mathrm{x}_{\mathrm{q}} . \quad \mathbf{n}$ is the normal vector to the crack surface, and $\mathbf{l}$ is the unit direction vector of the cack motion $b(y)$. S $(t)$ is the source-time function of crack kinetics and $\Delta \mathrm{V}$ is the crack volume.

Since eqn (1) is fairly complicated and contains two vectors $\mathbf{l}$ and $\mathbf{n}$, it is not suitable for an inverse problem. Thus, introducing moment tensor $\mathrm{M}_{\mathrm{pq}}$, eqn (1) is simplified as eqn (3),

$$
\begin{aligned}
& \int_{F} C_{p q k l} b(y) l_{k} n_{l} d F=C_{p q k l} l_{k} n_{1}\left[\int_{F} b(y) d F=C_{p q k l} l_{k} n_{1} \Delta V=M_{p q}\right. \\
& u_{k}(x, t)=G_{k p, q}(x, y, t) M_{p q} * S(t) .
\end{aligned}
$$

The moment tensor, $\mathrm{M}_{\mathrm{pq}}$, is defined by the product of the elastic constants $\left[\mathrm{N} / \mathrm{m}^{2}\right]$ and the crack volume $\left[\mathrm{m}^{3}\right]$, which leads to the moment as physical unit $[\mathrm{Nm}]$. In the case of an isotropic material,

$$
\mathrm{M}_{\mathrm{pq}}=\lambda \mathrm{l}_{\mathrm{k}} \mathrm{n}_{\mathrm{k}} \delta_{\mathrm{pq}}+2 \mu\left(\mathrm{l}_{\mathrm{p}} \mathrm{n}_{\mathrm{q}}+\mathrm{l}_{\mathrm{q}} \mathrm{n}_{\mathrm{p}}\right) \Delta \mathrm{V},
$$

where $\lambda$ and $\mu$ are Lame constants. $[6])$,

In damage mechanics (Kachanov [5]), damage tensor $\mathrm{D}_{\mathrm{pq}}$ is defined as (Ohtsu and Ohtsuka

$$
D_{p q}=\left(1_{p} n_{q}+l_{q} n_{p}\right) \Delta V /\left(2 V^{*}\right) .
$$

Here, $\mathrm{V}^{*}$ is the representative volume. Then, the scalar damage variable is derived ,

$$
\mathrm{D}=\mathrm{n}_{\mathrm{p}} \mathrm{D}_{\mathrm{pq}} \mathrm{n}_{\mathrm{q}}=\mathrm{l}_{\mathrm{k}} \mathrm{n}_{\mathrm{k}} \Delta \mathrm{V} / \mathrm{V}^{*} .
$$

From eqn (4), a trace component is obtained,

$$
\mathrm{M}_{\mathrm{kk}}=(3 \lambda+2 \mu) \mathrm{l}_{\mathrm{k}} \mathrm{n}_{\mathrm{k}} \Delta \mathrm{V} .
$$

Comparing eqn (7) with eqn (6), it is found that the trace component of the moment tensor is equivalent to the scalar damage variable. Consequently, damage evolution can be estimated from the summation of the trace components of the moment tensors. In addition, the accumulation of crack volumes is also relatively estimated from $\mathrm{M}_{\mathrm{kk}} / \mathrm{l}_{\mathrm{k}} \mathrm{n}_{\mathrm{k}}$.

\section{SiGMA PROCEDURE}

For the analysis of the inverse problem for eqn (3), the spatial derivatives of Green's functions are inevitably required. Accordingly, numerical solutions are obtained by FDM (Enoki et al. [7]) and by FEM (Hamstad et al. [8]). These solutions, however, need a vector processor for computation and are not readily applicable to processing a large amount of $\mathrm{AE}$ waves. Based on the far-filed term of $\mathrm{P}$ wave, a simplified procedure was developed, which is suitable for a PC-based processor and robust in computation. The procedure is now implemented as SiGMA (Simplified Green's functions for Moment tensor Analysis) code (Ohtsu [4]). 
By taking into account only the far field term of Green's functions in an infinite space, the displacement $U_{i}(\mathbf{x}, t)$ of $P$ wave motion is obtained from eqn (1),

$$
\mathrm{U}_{\mathrm{i}}(\mathbf{x}, \mathrm{t})=-1 /\left(4 \pi \rho v_{\mathrm{p}}^{3}\right) \mathrm{r}_{\mathrm{i}} \mathrm{r}_{\mathrm{p}} \mathrm{r}_{\mathrm{q}} / \mathrm{R} \mathrm{dS}(\mathrm{t}) / \mathrm{dt} \mathrm{M}_{\mathrm{pq}} \cdot
$$

Here $\rho$ is the density of the material and $v_{p}$ is P-wave velocity. $R$ is the distance between the source $\mathbf{y}$ and the observation point $\mathbf{x}$, of which direction cosine is $\mathbf{r}=\left(\mathrm{r}_{1}, \mathrm{r}_{2}, \mathrm{r}_{3}\right)$. Considering the effect of reflection at the surface and neglecting the source-time function, amplitude $\mathrm{A}(\mathbf{x})$ of the first motion is represented,

$$
\mathrm{A}(\mathbf{x})=\operatorname{Cs} \operatorname{Ref}(\mathbf{t}, \mathbf{r}) / \mathrm{R} r_{\mathrm{p}} \mathrm{M}_{\mathrm{pq}} \mathrm{r}_{\mathrm{q}}
$$

where Cs is the calibration coefficient including material constants in eqn (8). $\quad \mathbf{t}$ is the direction of the sensor sensitivity. $\operatorname{Ref}(\mathbf{t}, \mathbf{r})$ is the reflection coefficient at the observation location $\mathbf{x}$. Since the moment tensor is symmetric, the number of independent unknowns $\mathrm{M}_{\mathrm{pq}}$ to be solved is six. Thus, multi-channel observation of the first motions at more than six channels is required to determine the moment tensor components.

From AE waveform in Figure 1, two parameters of the arrival time (P1) and the amplitude of the first motion (P2) are determined. In the source location procedure, source location $\mathbf{y}$ is determined from these arrival time differences. Then, distance $\mathrm{R}$ and its direction vector $\mathbf{r}$ are determined. The amplitudes of the first motions at more than 6 channels are substituted into eqn 9, and the components of the moment tensor are determined. Since SiGMA code requires only relative values of the moment tensor components, the relative calibration of AE sensors is sufficient enough. Then, the classification of a crack is performed by the eigenvalue analysis of the moment tensor. Setting the ratio of the maximum shear contribution as $\mathrm{X}$, three eigenvalues for the shear crack become $\mathrm{X}, 0,-\mathrm{X}$. Likewise, the ratio of the maximum deviatoric tensile component is set as $\mathrm{Y}$ and the isotropic tensile as $\mathrm{Z}$. It is reasonably assumed that the principal axes of the shear crack is identical to those of the tensile crack. Then, the eigenvalues of the moment tensor for a general case are represented by the combination of the shear crack and the tensile crack. Thus, the eigenvalues are normalized and decomposed,

$$
\begin{array}{ll}
1.0 & =\mathrm{X}+\mathrm{Y}+\mathrm{Z}, \\
\text { the intermediate eigenvalue/the maximum eigenvalue } & =0-\mathrm{Y} / 2+\mathrm{Z}, \\
\text { the minimum eigenvalue/the maximum eigenvalue } & =-\mathrm{X}-\mathrm{Y} / 2+\mathrm{Z} .
\end{array}
$$

Here $\mathrm{X}, \mathrm{Y}$, and $\mathrm{Z}$ denote the shear ratio, the deviatoric tensile ratio, and the isotropic tensile ratio, respectively. In SiGMA code, AE sources of which the shear ratios are less than $40 \%$ are classified into tensile cracks. The sources of $\mathrm{X}>60 \%$ are classified into shear cracks. In between $40 \%$ and $60 \%$, cracks are referred to as mixed mode.

\section{MOMENT TENSOR AND MIXED-MODE CRACKING}

Erdgan and Sih [9] proposed the maximum circumferential stress criterion for the mixed-mode crack extension. As given in Figure 2, the direction of crack extension to a pre-existing crack is determined as the direction of the maximum circumferential stress,

$$
\mathrm{K}_{\mathrm{I}} \sin \theta+\mathrm{K}_{\mathrm{II}}(3 \cos \theta-1)=0
$$




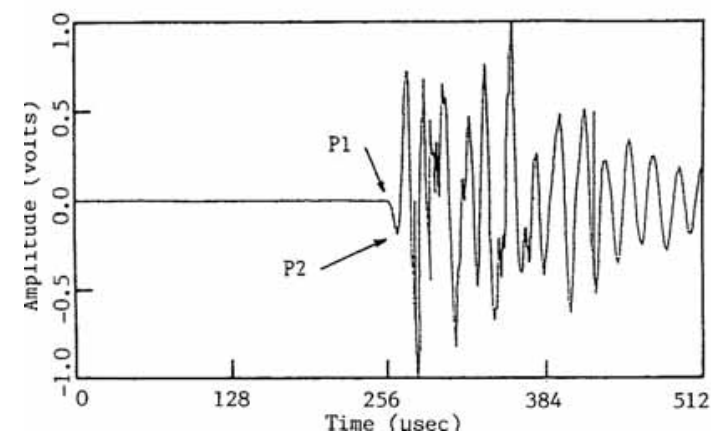

Figure 1: AE waveform.

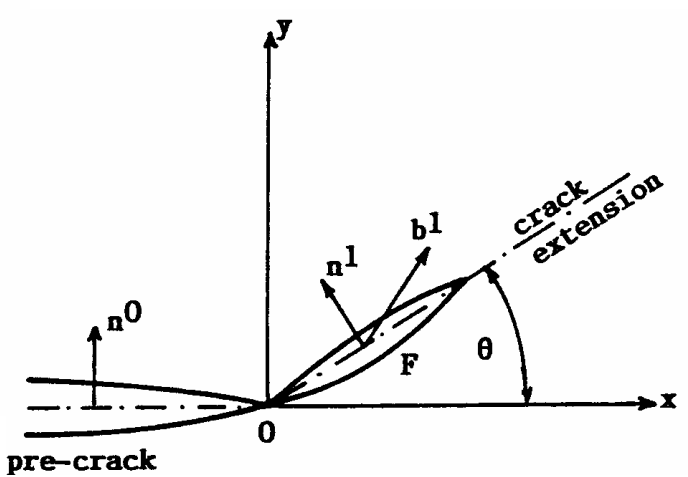

Figure 2: Crack extension from a pre-crack.

Where $\mathrm{K}_{\mathrm{I}}$ and $\mathrm{K}_{\mathrm{II}}$ are the stress intensity factors of mode I and mode II, respectively. Initiation of the crack is governed by,

$$
\cos \theta / 2\left[\mathrm{~K}_{\mathrm{I}} \cos ^{2} \theta / 2-3 / 2 \mathrm{~K}_{\mathrm{II}} \sin \theta\right]=\mathrm{K}_{\mathrm{IC}} .
$$

Here $\mathrm{K}_{\mathrm{IC}}$ is the critical stress intensity factor. From eqns (11) and (12), normalized stress intensity factors $\mathrm{K}_{\mathrm{I}}^{*}=\mathrm{K}_{\mathrm{I}} / \mathrm{K}_{\mathrm{IC}}$ and $\mathrm{K}_{\mathrm{II}} *=\mathrm{K}_{\mathrm{II}} / \mathrm{K}_{\mathrm{IC}}$ are derived as,

$$
\mathrm{K}_{\mathrm{I}}^{*}=(3 \cos \theta-1) /[\cos \theta / 2(\cos \theta+1)], \text { and } \mathrm{K}_{\mathrm{II}} *=-\sin \theta /[\cos \theta / 2(\cos \theta+1)]
$$

In the eigenvalue analysis, three eigenvectors are also determined, and then the vectors $\mathbf{I}$ and $\mathbf{n}$, which are interchangeable, are recovered. Three eigenvectors e1, e2, and $\mathbf{e} 3$ are obtained as,

$$
\begin{aligned}
& \mathbf{e} 1=\mathbf{l}+\mathbf{n} \\
& \mathbf{e} 2=\mathbf{l} \times \mathbf{n} \\
& \mathbf{e} 3=\mathbf{l}-\mathbf{n} .
\end{aligned}
$$

Here $\mathrm{x}$ denotes the vector product. From these relations, the two vectors $\mathbf{I}$ and $\mathbf{n}$ associated with the orientation of a crack can be recovered. According to Figure $2, \cos \theta=\mathbf{n}^{\mathbf{0}} \mathbf{n}^{\mathbf{1}}$, and $\mathbf{n}^{\mathbf{1}}$ is identical to vector $\mathbf{n}$ in eqn (14). Consequently, normalized stress intensity factors in eqn (13) can be determined experimentally from SiGMA analysis (Ohtsu et al. [10]).

\section{MICROCRAKING PROCESS IN FRACTURE PROCESS ZONE}

In a three-point bending test of an off-centered-notched concrete beam, SiGMA analysis was performed. Results are given in Figure 3. The tensile crack is indicated by arrow symbol, while the shear crack is denoted by cross symbol. The directions of two vectors $\mathbf{l}$ and $\mathbf{n}$ are shown in their directions. It is found that both types of tensile cracks and shear cracks are observed in the fracture process zone ahead of the notch. Then, damage evolution was estimated by eqn (7). It is realized that the evolution process is fairly gradual, nucleating all types of tensile, shear and mixed-mode cracks.

To investigate the microcracking process, analysis of the two-domain boundary element 


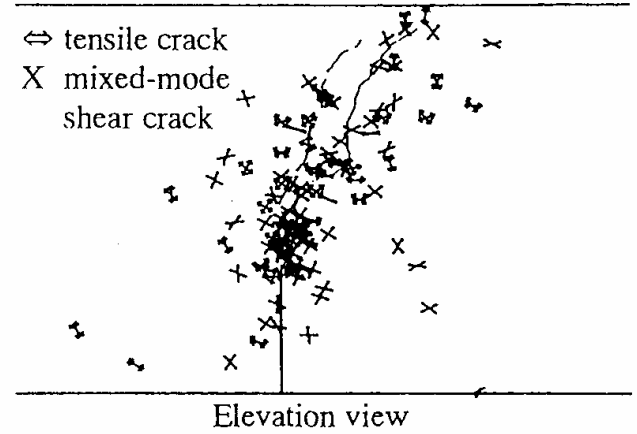

Figure 3: Results of SiGMA analysis.

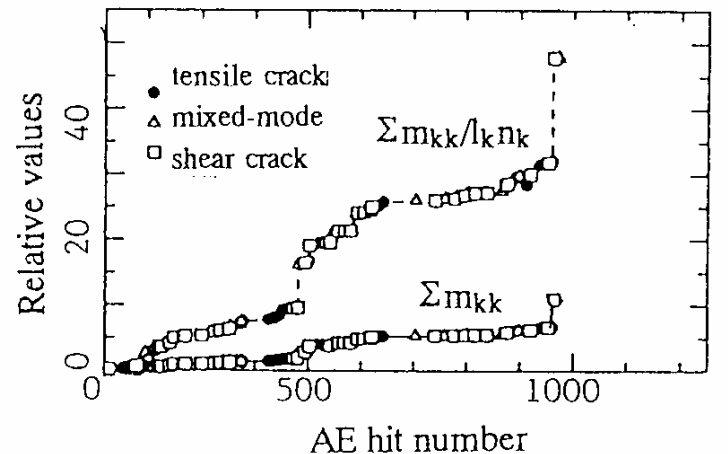

Figure 4: Evolution of damage in the process zone.

method (BEM) was performed. Crack traces observed in the test and the analysis are compared in Figure 5. It is found that the crack trace is reasonably simulated by BEM.

Normalized stress intensity factors (SIF) are then calculated from results of SiGMA analysis and are shown in Figure 6. These are plotted against AE hit number. In the beginning stage of microcraking process, $\mathrm{K}_{\mathrm{I}}{ }^{*}$ is dominant. Then, $\mathrm{K}_{\mathrm{II}}{ }^{*}$-dominant cracks are observed in the final stage. In order to confirm this result, normalized SIFs were determined from

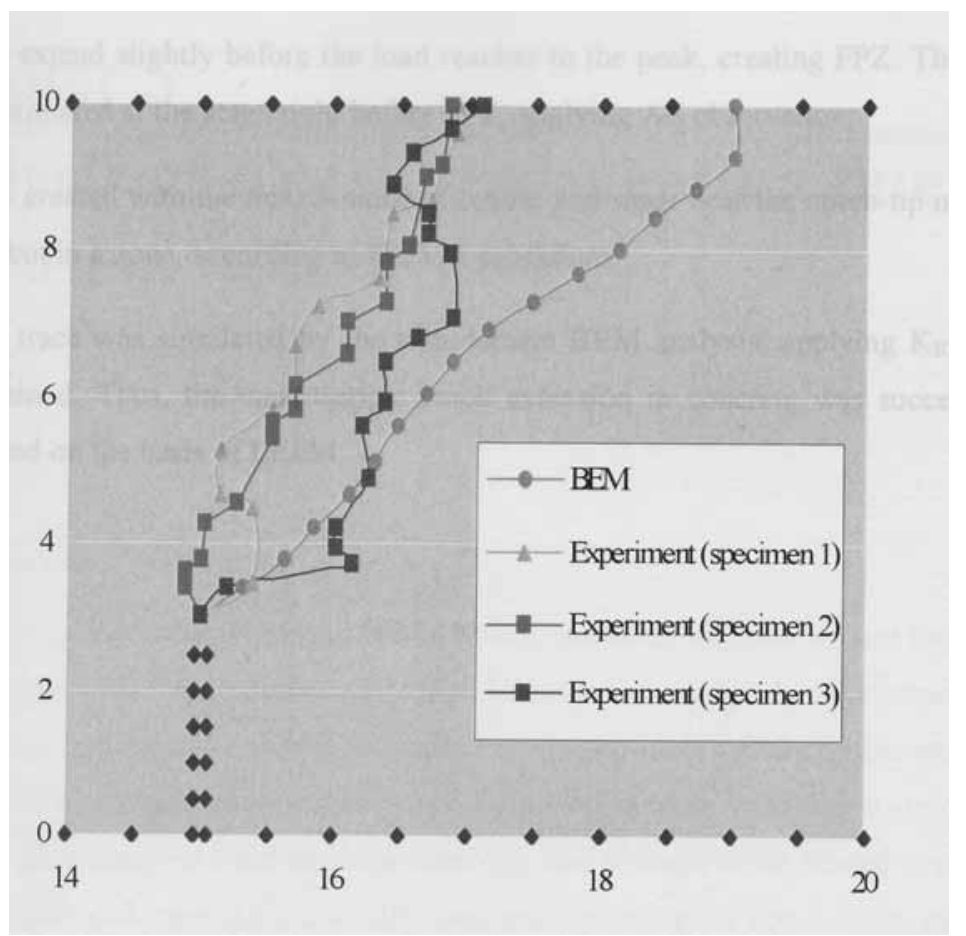

Figure 5: Crack traces observed in the test and analyzed by BEM. 
BEM analysis. Results are shown in Figure 7. Under crack extension, $\mathrm{K}_{\mathrm{I}}$ * decreses and $\mathrm{K}_{\mathrm{II}}{ }^{*}$ increases. Thus, the tendency observed in SiGMA analysis is demonstrated. It is concluded that the moment tensor analysis is very promising to quantitatively identify microcracking processes in the fracture process zone of concrete.

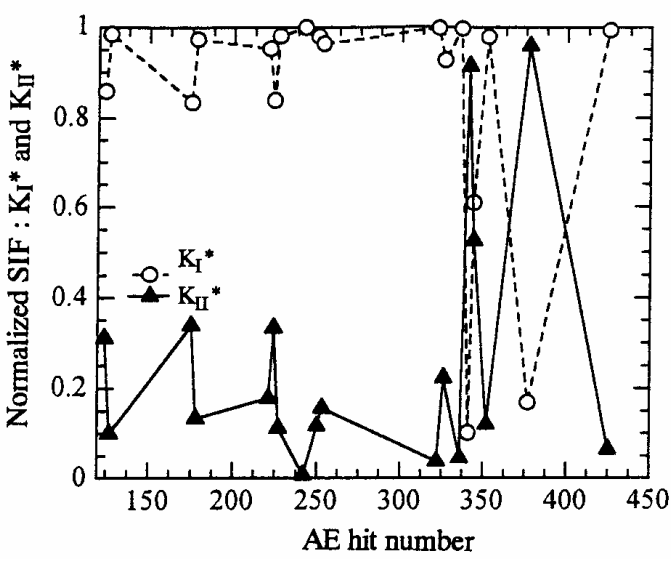

Figure 6: Normalized SIF by SiGMA analysis.

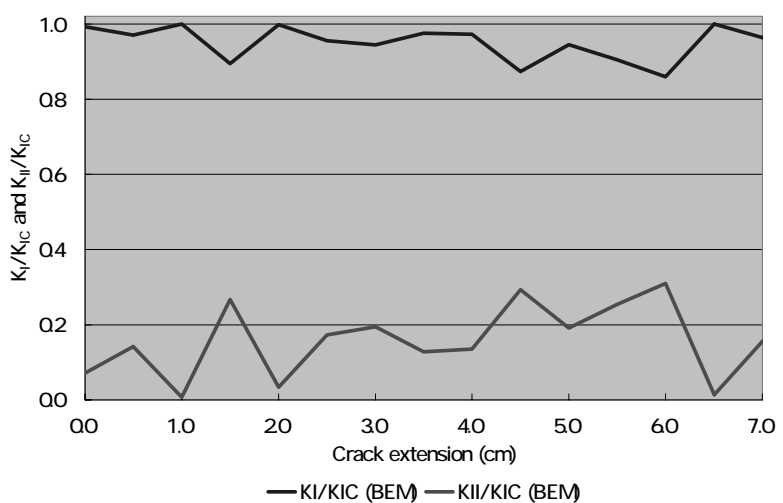

Figure 7: Normalized SIF by BEM analysis.

\section{REFERENCES}

1. Ohtsu, M. and Ono, K. "A Generalized Theory of Acoustic Emission and Green's Functions in a Half Space," Journal of AE, Vol. 3, No. 1, 124-133, 1984.

2. Ohtsu, M."Source Mechanism and Waveform Analysis of Acoustic Emission in Concrete," Journal of AE, Vol. 2, No. 1, 103-112, 1982.

3. Ohtsu, M., Okamoto, T. and Yuyama, S,"Moment Tensor Analysis of Acoustic Emission for Cracking Mechanisms in Concrete,” ACI Structural Journal, Vol. 95, No. 2, 87-95, 1998.

4. Ohtsu, M.,"Acoustic Emission Theory for Moment Tensor Analysis," Research in Nondestructive Evaluation, No. 6, 169-184, 1995.

5. Kachanov, M.'Effective Elastic Properties of Cracked Solids," Applied Mechanics Review, Vol. 45, No. 8, 304-335, 1992.

6. Ohtsu, M. and Ohtsuka, M."Damage Evolution by AE in the Fracture Process Zone of Concrete,” J. Materials, Conc. Struct. Pavement, JSCE, No. 599/V-40, 177-184, 1998.

7. Enoki, M., Kishi, T. and Kohara, S."Determination of Micro-cracking Moment Tensor of Quasi-cleavage Facet by AE Source Characterization," Progress in Acoustic Emission III, JSNDI, 763-770, 1986.

8. Hamstad, M. A., O'Gallagher, A. and Gary, J.'Modeling of Buried Monopole and Dipole Source of Acoustic Emission with a Finite Element Technique, Journal of AE, Vol. 17, Nos. 3-4, 97-110, 1999.

9. Erdogan, F. and Sih, G. C.”On the Crack Extension in Plates under Plane Loading and Transverse Shear, “ J. Basic Engineering, Transactiuons of ASME, No. 12, 519-527, 1963.

10. Ohtsu, M., Munwam, M. C. and Rossmanith, H. P.”Crack Extension in Cementitious Materials by AE," Concrete Science and Engineering, Vol. 1, 157-165, 1999. 